\title{
(2) REVIEW
}

(อ)

OPEN ACCESS

Evidence-based prevention of Alzheimer's disease: systematic review and meta-analysis of 243 observational prospective studies and 153 randomised controlled trials

\author{
Jin-Tai Yu $\odot,{ }^{1}$ Wei Xu $\odot,{ }^{2}$ Chen-Chen Tan, ${ }^{2}$ Sandrine Andrieu, ${ }^{3}$ John Suckling, ${ }^{4}$ \\ Evangelos Evangelou, ${ }^{5}$ An Pan, ${ }^{6}$ Can Zhang, ${ }^{7}$ Jianping Jia, ${ }^{8}$ Lei Feng, ${ }^{9}$ Ee-Heok Kua, ${ }^{9}$ \\ Yan-Jiang Wang, ${ }_{10}$ Hui-Fu Wang, ${ }^{2}$ Meng-Shan Tan, ${ }^{2}$ Jie-Qiong Li, ${ }^{2}$ Xiao-He Hou, ${ }^{2}$ \\ Yu Wan, ${ }^{2}$ Lin Tan, ${ }^{2}$ Vincent Mok, ${ }_{11}^{11}$ Lan Tan, ${ }^{2}$ Qiang Dong, ${ }_{1}^{1}$ Jacques Touchon, ${ }^{12}$ \\ Serge Gauthier, ${ }^{13}$ Paul S Aisen, ${ }^{14}$ Bruno Vellas ${ }^{15}$
}

- Additional material is published online only. To view please visit the journal online (http://dx.doi.org/10.1136/ jnnp-2019-321913).

For numbered affiliations see end of article.

\section{Correspondence to}

Professor Jin-Tai Yu, Department of Neurology and Institute of Neurology, Huashan Hospital, Shanghai Medical College, Fudan University, Shanghai 200040,China; jintai_yu@

fudan.edu.cn

J-TY, WX and C-CT contributed equally.

Received 22 August 2019 Revised 26 February 2020 Accepted 22 April 2020 Published Online First 20 July 2020

\section{SLinked}

- http://dx.doi.org/10.1136/ jnnp-2020-323606

Check for updates

(C) Author(s) (or their employer(s)) 2020. Re-use permitted under CC BY-NC. No commercial re-use. See rights and permissions. Published by BMJ.

To cite: Yu J-T, Xu W, Tan C-C, et al. J Neurol Neurosurg Psychiatry

2020:91:1201-1209.

\section{ABSTRACT}

Background Evidence on preventing Alzheimer's disease (AD) is challenging to interpret due to varying study designs with heterogeneous endpoints and credibility. We completed a systematic review and metaanalysis of current evidence with prospective designs to propose evidence-based suggestions on $A D$ prevention. Methods Electronic databases and relevant websites were searched from inception to 1 March 2019. Both observational prospective studies (OPSs) and randomised controlled trials (RCTs) were included. The multivariableadjusted effect estimates were pooled by random-effects models, with credibility assessment according to its risk of bias, inconsistency and imprecision. Levels of evidence and classes of suggestions were summarised.

Results A total of 44676 reports were identified, and 243 OPSs and 153 RCTs were eligible for analysis after exclusion based on pre-decided criteria, from which 104 modifiable factors and 11 interventions were included in the meta-analyses. Twenty-one suggestions are proposed based on the consolidated evidence, with Class I suggestions targeting 19 factors: 10 with Level A strong evidence (education, cognitive activity, high body mass index in latelife, hyperhomocysteinaemia, depression, stress, diabetes, head trauma, hypertension in midlife and orthostatic hypotension) and 9 with Level B weaker evidence (obesity in midlife, weight loss in late life, physical exercise, smoking, sleep, cerebrovascular disease, frailty, atrial fibrillation and vitamin C). In contrast, two interventions are not recommended: oestrogen replacement therapy (Level A2) and acetylcholinesterase inhibitors (Level B).

Interpretation Evidence-based suggestions are proposed, offering clinicians and stakeholders current guidance for the prevention of $A D$.

\section{INTRODUCTION}

An unequivocal downtrend in the prevalence and incidence of dementia was recently reported and associated with earlier population-level investment (eg, improved education and vascular health), ${ }^{1-3}$ strengthening the necessity for primary prevention. ${ }^{4}$
The past few decades have witnessed great global efforts in updating and upgrading the evidence on how to prevent Alzheimer's disease (AD), ${ }^{5} 6$ accounting for approximately two-thirds of all cases of dementia and affecting up to $20 \%$ of individuals older than 80 years. ${ }^{78}$ Nevertheless, key issues in the field are the inconsistency among conclusions and variable levels of credibility arising from the wide variety of study designs. ${ }^{9}$ Two types of studies are generally regarded as having the greatest impact on the extant literature: (1) observational prospective studies (OPSs), which describe temporal relationships with potential causal links and often use large samples recruited from community dwellers; and (2) randomised controlled trials (RCTs), which possess strong internal validity to infer causality by testing the effects of specific interventions on the incidence of $\mathrm{AD}$. Although both approaches are useful, the major concerns in OPSs are usually the elusive sources of bias when interpreting the identified wide-ranging factors, and current RCTs are often compromised by short follow-up durations, subjective endpoints, small sample sizes and specific recruitment criteria with uncertain generalisability. ${ }^{5}$

Considerable evidence has been generated regarding AD through OPSs and RCTs. Because it is almost impossible to conduct RCTs that evaluate all risk factors of $\mathrm{AD}$, a quantitative depiction of $\mathrm{AD}$ 's prevention 'profile' based on these two complementary study types is urgently needed for prevention guidelines that weigh the benefits against the risks. Deconstructing the bias sources from OPSs will facilitate the interpretation of credibility ratings and also guide future research directions. In this study we consolidated the extant evidence from both OPSs and RCTs to formulate the levels of evidence and classes of clinical suggestions for $\mathrm{AD}$ prevention.

\section{METHODS}

Search strategy and selection criteria

We followed the recommendations of the Preferred Reporting Items for Systematic reviews and MetaAnalyses (PRISMA) 2009 guidelines. ${ }^{10} 11$ PubMed, 


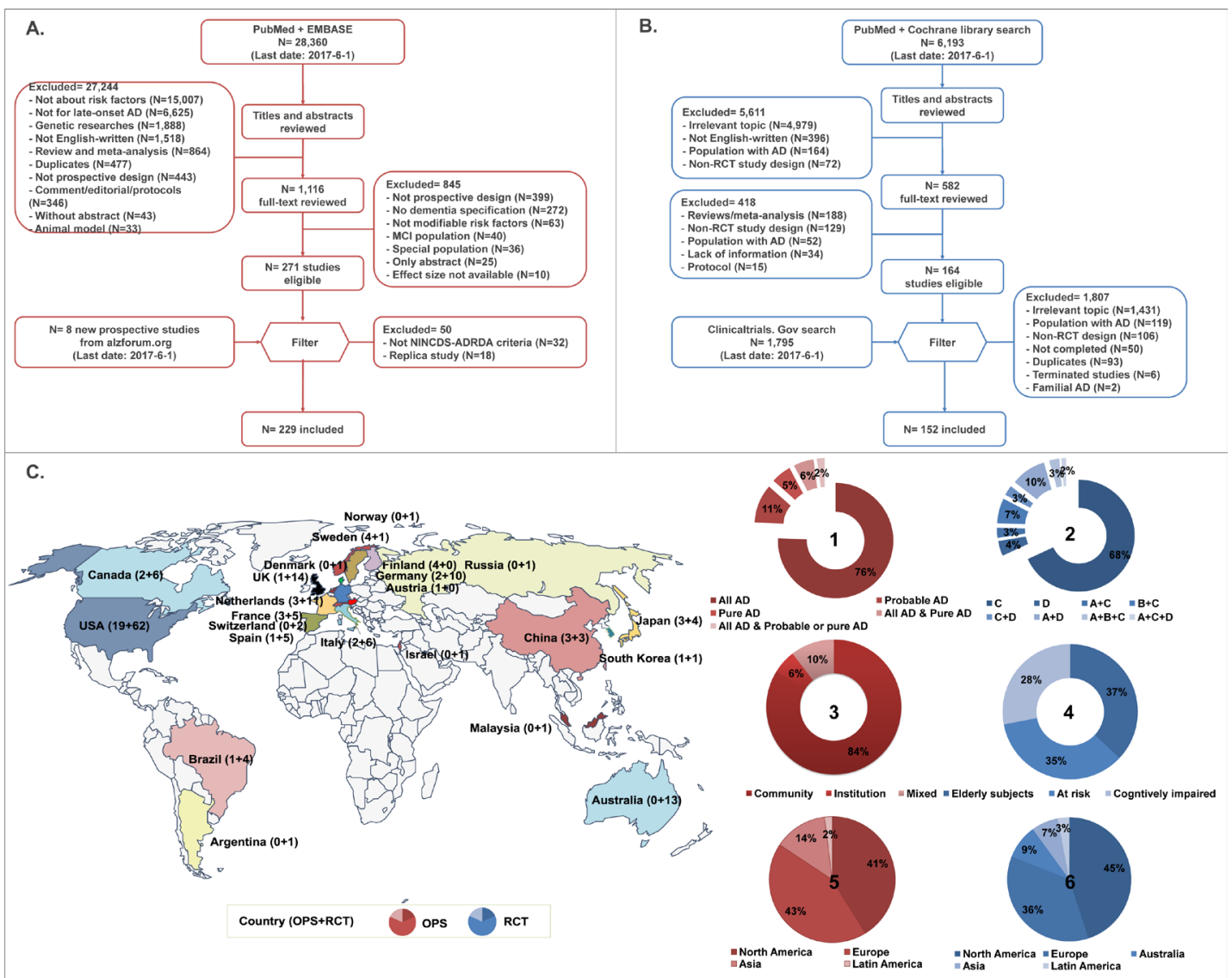

Figure 1 Flow chart showing literature selection for OPSs (figure 1A) and RCTs (figure 1B) and map depicting studies eligible for systematic review (figure 1C). A total of 243 OPSs (figure 1A) and 153 completed RCTs (figure 1B) were finally included. 243 OPSs from 17 countries on four continents (Europe accounting for 43\%, North America 41\%, Asia 14\% and Latin America 2\%) reported the association of 134 modifiable risk factors with risk of clinical Alzheimer's-type dementia (83\% used all AD, 13\% probable AD and 11\% pure AD) diagnosed by NINCDS-ADRDA criteria in populations with various racial backgrounds (68\% white, 14\% Asian descent, 13\% mixed race), sources ( $84 \%$ community, $6 \%$ institution, $10 \%$ mixed source) and baseline cognitive statuses ( $82 \%$ free of dementia, 16\% cognitively normal, $2 \%$ unclear). A total of 153 published RCTs from five continents (North America accounting for 45\%, Europe 36\%, Australia 9\%, Asia 7\% and Latin America 3\%) reported the effects of 15 types of interventions on AD (7\%), dementia $(16 \%)$ and cognitive function (85\%) in selected participants, including elderly subjects (37\%), high-risk group (35\%) or cognitively impaired (28\%) (figure $1 \mathrm{C}$ ). In the pie charts, 1 and 2 show the outcome (all $A D=$ probable or possible $A D$, or $A D$ with or without VD/CVD, Pure $A D=A D$ without VD or CVD; $A=$ Alzheimer's disease, $B=$ Biomarker of $A D, C=$ Cognition, $D=$ Dementia); 3 and 4 show the population source; 5 and 6 show the percentage of studies from different continents. AD, Alzheimer's disease; CVD, cerebrovascular disease; OPS, observational prospective study; RCT, randomised controlled trial; VD, vascular dementia.

EMBASE and CENTRAL were searched using the terms "Alzheimer's", "Alzheimer", "dementia", and "risk" for OPS and "Alzheimer", “cognitive”, "cognition”, “prevent”, and "prevention" for RCT up to 1 March 2019. Bibliographies of relevant literature and records in Clinicaltrials.gov and AlzRisk database $^{12}$ were hand-searched in case of omission. The inclusion criteria were as follows: (1) an OPS exploring the association between potentially modifiable exposures at baseline and incident $\mathrm{AD}$ independently diagnosed according to the National Institute of Neurological and Communicative Disorders and Stroke and the Alzheimer's Disease and Related Disorders Association (NINCDS-ADRDA) criteria, ${ }^{13}$ or (2) a RCT targeting the impact of addressing modifiable risk factors on the incidence of $\mathrm{AD}$ or $\mathrm{AD}$-related clinical endpoints (dementia or cognitive impairment), and (3) a publication written in English to permit easy access to the source information of all included articles. The detailed exclusion criteria are shown in figure 1. Bibliographies of relevant original studies and systematic reviews were handsearched. Literature selection was performed by three pairs of experienced investigators (JTY, WX, CCT, HFW, MST and JQL) and any disagreements on inclusion were resolved by consensus and arbitration by a panel of investigators within the review team (JTY, WX, CCT, HFW, MST, JQL and Lan Tan).

\section{Data extraction}

Pre-designed templates were used to extract the data with reference to the STROBE statement (https:/www.equator-network. org/reporting-guidelines/strobe/). An evidence-based profile of AD modifiable risk factors was established for better tracing of bias sources. The multivariable-adjusted risk estimates were extracted. If these estimates were unavailable, we attempted to obtain them by contacting the corresponding authors. The stringently performed process comprised three independent steps: (a) data extraction by three pairs of experienced investigators (JTY, WX, CCT, HFW, MST and JQL); (b) independent data proof reading by 10 researchers (JTY, WX, CCT, HFW, MST, JQL, XHH, YW, Lin Tan and Lan Tan); and (c) addressing discrepancies by consensus and arbitration. 


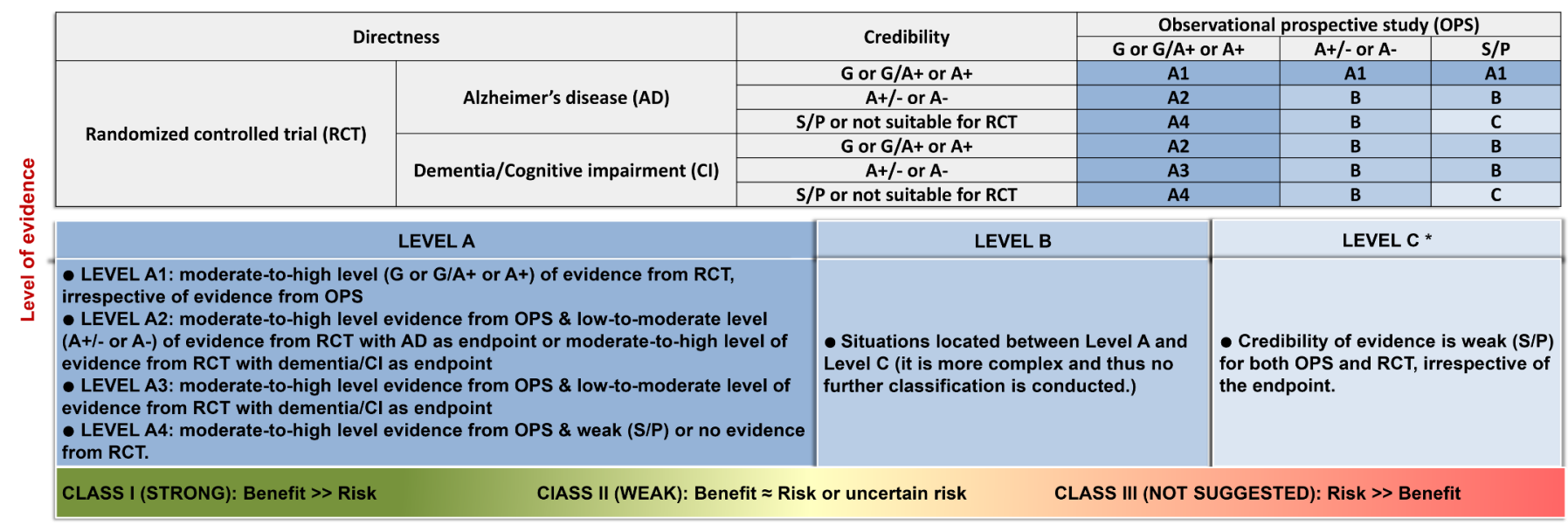

Class (STRENGTH) of suggestions

Figure 2 Rating levels of evidence and strength of suggestions. Based on the Cochrane Handbook for Systematic Reviews of Interventions for RCTs and the Newcastle-Ottawa Quality Assessment Scale (NOS) for OPSs, we evaluated the quality of eligible studies. The credibility of each result was then categorised into four levels: Good (G level), Acceptable ( $A_{ \pm}$level), Susceptible ( $S_{ \pm}$level) and Poor (P level) according to the score combination of three domains: risk of bias, inconsistency and imprecision. Levels of evidence were summarised, representing the quality of scientific evidence on the basis of directness of outcome (for RCTs), consistency and quality of data from clinical trials and/or observational studies. Classes of suggestions were made after weighing the benefits against the risks due to specific interventions. *Factors rated with 'level $C^{\prime}$ evidence were not considered for recommendation in the present study.

Assessment of study quality and credibility of meta-analyses The risk of bias tool proposed by Cochrane ${ }^{14}$ for RCTs and involving the Newcastle-Ottawa Quality Assessment Scale $(\mathrm{NOS})^{15}$ for OPSs were used to evaluate the quality of eligible studies. The total score for the Cochrane tool or NOS was regarded as a proxy to assess the overall risk of bias for each single study. The score for each item evaluated the associated risk of bias (online supplementary appendix 1). The credibility of each meta-analysis result was then categorised into four levels: Good (G level), Acceptable (A \pm level), Susceptible ( $\mathrm{S} \pm$ level) and Poor (P level) according to the score combination of three domains: risk of bias, ${ }^{16}$ inconsistency ${ }^{17}$ and imprecision ${ }^{18}$ (online supplementary appendix 2). In particular, $G$ and $A+$ levels were regarded as moderate-to-high credibility.

\section{Levels of evidence and strength of suggestions}

Levels of evidence were summarised to represent the quality of scientific evidence on the basis of directness of outcome for $\mathrm{AD}$, credibility of meta-analyses and consistency of evidence from clinical trials and/or observational studies: Level $\mathrm{A}>$ Level $\mathrm{B}>$ Level C (based on the evidence level). Classes of recommendations were made after weighing the benefits against the risks due to specific interventions: Class I (strong recommendation), Class II (weak recommendation) and Class III (not recommended) (figure 2).

\section{Statistical analyses}

The multivariable-adjusted risk estimates and 95\% confidence intervals (CI) were log-transformed and combined using random models (DerSimonian-Laird method). ${ }^{19}$ Sensitivity analyses excluding odd ratios (ORs) reported by some OPSs were performed because ORs tend to overestimate the effect size compared with the relative risk (RR), particularly when the incidence is not small. A 95\% prediction interval (PI) was calculated to better evaluate the precision of the result. ${ }^{20}$ Heterogeneity was assessed by $\mathrm{Q}$ test and quantified by the $I^{2}$ metric. ${ }^{21}$ The source of heterogeneity was explored via sensitivity analyses, meta-regression and subgroup analyses. The robustness of the results was examined by excluding those rated as at a higher risk of bias. Publication bias was assessed following two steps: (1) testing the symmetry of the funnel plot by the Egger method ${ }^{22}$; and (2) determining whether any asymmetry was due to publication bias via enhanced-contour funnel plots after the trimand-fill method. ${ }^{23}$ The meta-regression and publication bias test were conducted only when at least 10 studies were available. The "metagen", "metabias" and "trimfill" packages in R 3.4.3 software (https://www.r-project.org) were used to perform all the analyses.

Additionally, multiple subgroup and sensitivity analyses were conducted to take into account the following cases where results might be biased. First, $82 \%$ of studies recruited people without dementia at baseline and only $17 \%$ specifically constrained the population to those with normal cognition. Notably, inclusion of individuals with mild cognitive impairment, who might be at a prodromal stage of $\mathrm{AD}$, resulted in a degree of misclassification bias, especially when the population was at an advanced age and was insufficiently followed. Thus, subgroup analyses according to the cognitive status at baseline (free of dementia vs cognitively normal), sufficiency of follow-up (online supplementary appendix 1) and life stage were performed. Second, it was often clinically difficult to distinguish mixed $\mathrm{AD}$ (coexistence of $\mathrm{AD}$ and vascular dementia (VD)) from VD among elderly people, especially when the pathological evidence is often unavailable and the individual has a history of stroke. Thus, to examine the influence of potential misclassification bias, subgroup analyses based on $\mathrm{AD}$ outcomes (all $\mathrm{AD}$ vs probable or pure $\mathrm{AD}(\mathrm{p}-\mathrm{AD})$ defined as $\mathrm{AD}$ without $\mathrm{VD}$ or cerebrovascular disease (CVD)) were performed. Third, sensitivity analyses excluding studies with high attrition rates and poor generalisability (online supplementary appendix 1) were conducted.

\section{Patient involvement}

No patients were involved in setting the research question or the outcome measures, nor were they involved in developing plans for design or implementation of the study. No patients were asked to advise on interpretation or writing up of results. There 

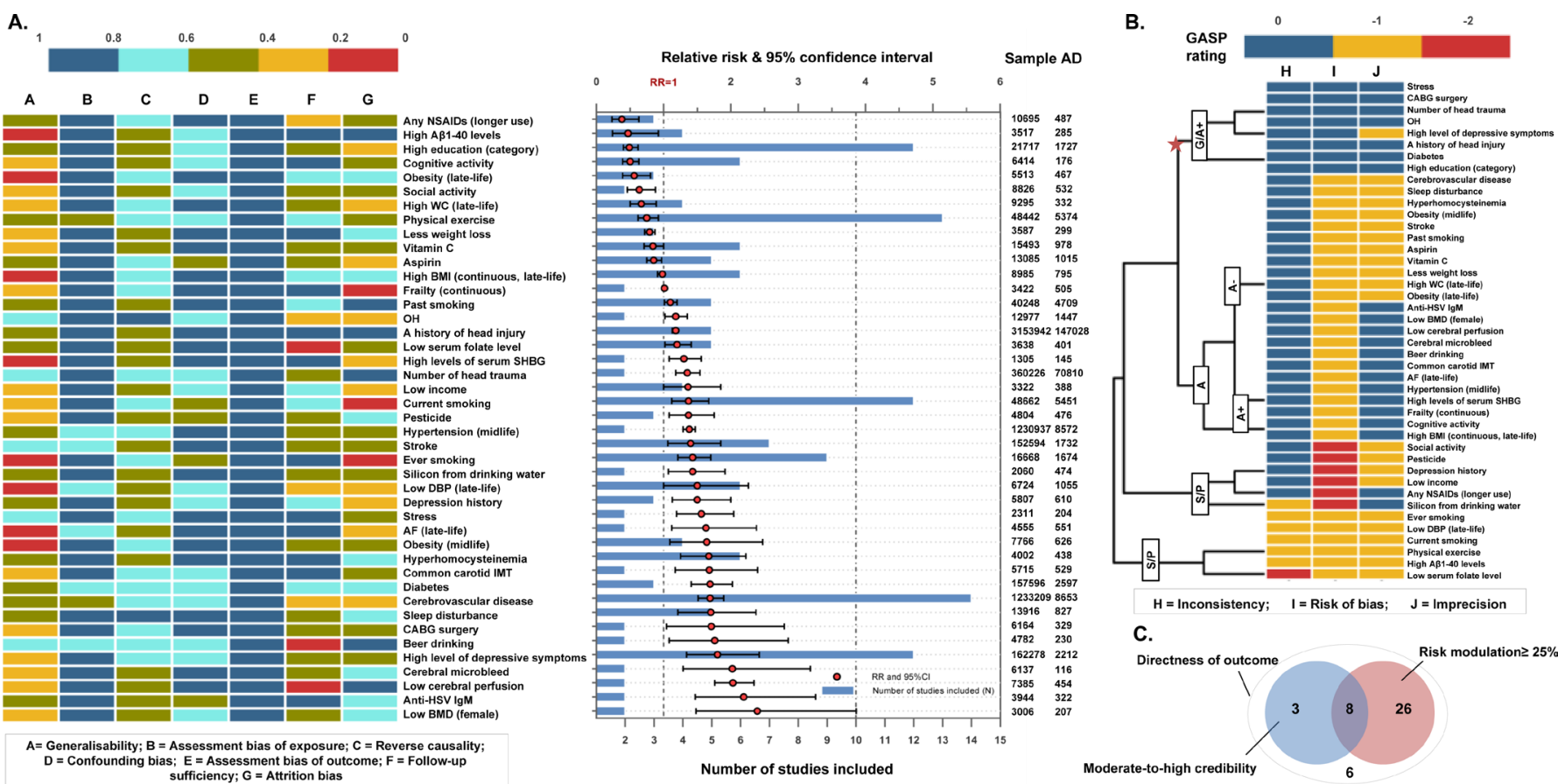

Figure 3 Risk of bias profile, meta-analysis results, sample size (figure 3A), credibility rating (figure 3B) and summary (figure 3C) for 43 significant modifiable risk factors based on observational prospective studies. When the mean score (for each bias domain) $\leq 0.5$ was regarded as possibly moderateto-high risk, analyses for $79 \%$ of factors had problems of generalisability, $60 \%$ for high attrition, $48 \%$ for insufficient follow-up, $40 \%$ for reverse causality, $8 \%$ for confounding bias and $6 \%$ for assessment of exposure. For a summary of the effect, a total of 43 factors showed significant associations with AD risk; 26 risk factors and eight protective factors were identified that modify the risk by at least $25 \%$ (figure $3 \mathrm{~A}$ ). For credibility of the pooled results, 11 factors were rated at a moderate-to-high level $(G, G / A+$ or $A+$ level), 20 were rated at a low-to-moderate level $(A+/ A-$ or $A$ - level) and 12 were rated at a very low level (S+, $\mathrm{S}$ - or P level) (figure 3B). With good performance in all the domains above, eight risk factors are highlighted (figure 3C). AD, Alzheimer's disease; BMD, bone mineral density; BMI, body mass index; CVD, cerebrovascular disease; DBP, diastolic blood pressure; HSV, herpes simplex virus; IMT, intima-media thickness; NSAIDs, non-steroidal anti-inflammatory drugs; SHBG, sex hormone binding globulin.

are no plans to disseminate the results of the research to study participants or the relevant patient community. No evaluation was undertaken to determine whether the studies included in the review had any patient involvement.

\section{RESULTS}

Figure 1 shows the flow diagrams of the study selection process for OPSs (figure 1A) and RCTs (figure 1B). The search yielded 33145 and 11531 records for OPSs and RCTs, respectively. After integration with the AlzRisk database and Clinicaltrials. gov website, a total of 243 OPSs and 153 completed RCTs were finally included. Evidence-based profiles were constructed (online supplementary appendix $3 \& 4$ ). The global distribution of studies eligible for the systematic review and their characteristics are shown in figure $1 \mathrm{C}$. The sources of bias for the current evidence profile mainly consisted of generalisability, attrition and misclassification for OPSs and performance bias, incomplete outcome data, inadequate allocation concealment and selective outcome reporting for RCTs (online supplementary appendix figure 1).

Meta-analyses were conducted for 134 risk factors (online supplementary appendix 5). A total of 43 factors showed significant associations with the risk of $\mathrm{AD}$, among which $80 \%$ were identified as significantly modifying the risk by at least $25 \%$ (figure 3A). Indicating the credibility of pooled results, analyses for eight risk factors (diabetes, orthostatic hypotension, hypertension in midlife, head trauma, stress, depression, midlife obesity and coronary artery bypass grafting (CABG) surgery) and three protective factors (cognitive activity, increased BMI in late life and education) were rated with moderate-to-high level credibility $(G, G / A+$ or $A+$ level). In addition, 20 factors were rated at a low-to-moderate level $(\mathrm{A}+/ \mathrm{A}-$ or $\mathrm{A}-$ level) and 12 were rated at a very low level $(\mathrm{S}+, \mathrm{S}-$ or $\mathrm{P}$ level) (figure $3 \mathrm{~B})$. With good performance in all the domains above, eight factors were highlighted, including depression (A+ level; RR 1.80; 95\% CI 1.34 to 2.42$)$, CABG surgery (G/A+ level; RR $1.71 ; 95 \% \mathrm{CI}$ 1.04 to 2.79 ), diabetes mellitus (G level; RR 1.69; 95\% CI 1.51 to 1.89 ), stress (G/A+ level; RR 1.56 ; $95 \% \mathrm{CI} 1.19$ to 2.04$)$, hypertension in midlife (G/A+ level; RR 1.38; 95\% CI 1.29 to 1.47), head trauma (G/A+ level; RR 1.35 ; $95 \%$ CI 1.18 to 1.54$)$, cognitive activity (A+ level; RR $0.50 ; 95 \%$ CI 0.39 to 0.63$)$ and more formal schooling years ( $>6$ to 15 years) (G level; RR 0.49; $95 \%$ CI 0.40 to 0.62 ) (figure 3C). Additionally, another 91 items were found to impart no influence on the risk of $\mathrm{AD}$, but mostly with low levels of credibility, except for late-life hypertension (G level, RR 0.96 ; 95\% CI 0.79 to 1.17 ) (online supplementary appendix figure 2).

For RCTs, 29 meta-analyses covering 11 interventions were conducted (online supplementary appendix 6). Three interventions, including total homocysteine ( $\mathrm{tHcy}$ )-lowering treatment (using folic acid, vitamin B12 and vitamin B6), cocoa flavanol and physical activity showed significant associations with $\mathrm{AD}$ or cognitive endpoints. For the directness of the outcomes, only five meta-analyses (involving acetylcholinesterase inhibitor, antihypertensive treatment, non-steroidal anti-inflammatory drugs (NSAIDs), hormone replacement therapy and ginkgo biloba) examined associations with $\mathrm{AD}$ (figure $4 \mathrm{~A}$ ). For the levels of credibility, nine meta-analyses were rated at a moderate-to-high level (G, G/A+ or 

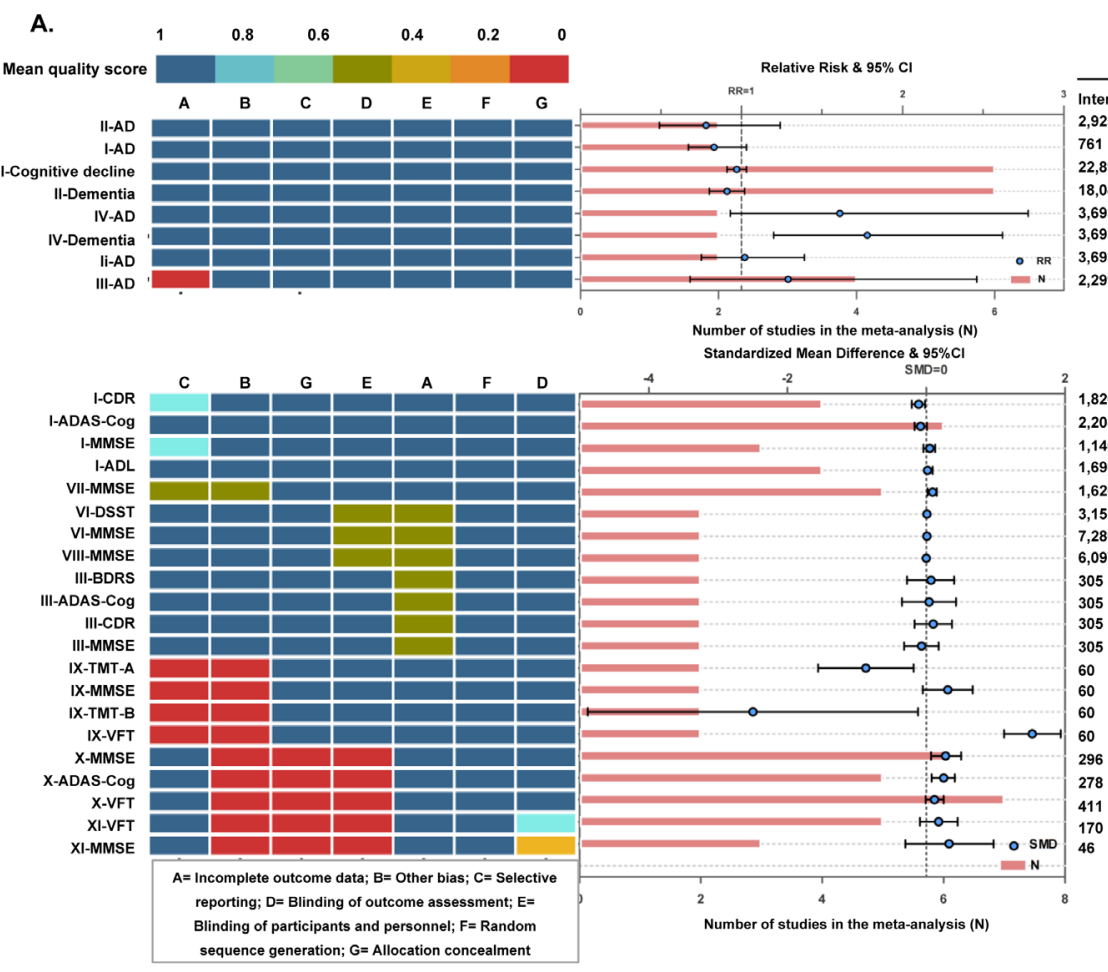

L. Acetylcholinesterase inhibitor; II. Anti-hypertensive agents in late-life; III. NSAIDs; IV. Hormone therapy; V. Ginkgo biloba; VI. Glycemic control; VII. Homocysteine-lowering treatment; VIII. DHA+EPA; IX. Cocoa flavanol; X. Physical exercise XI. Cognitive training

Figure 4 Risk of bias profile, meta-analysis results, sample size (figure 4A), credibility rating (figure 4B) and summary (figure 4C) for 11 interventions based on randomised controlled trials. When the mean score (for each bias domain) $\leq 0.5$ was regarded as possibly moderate-to-high risk, $17.2 \%$ metaanalyses had problems of inadequate concealment of allocations, $27.6 \%$ for performance bias, 3.4\% for detection bias, $24.1 \%$ for incomplete outcome data, $13.8 \%$ for selective outcome reporting and $31 \%$ for other sources of bias. For the significance of the pooled results, six meta-analyses showed significant associations (figure 4A). For credibility of the pooled results, nine meta-analyses were rated at a moderate-to-high level (G, G/A+ or A+ level), three at a low-to-moderate level (A+/A- or $\mathrm{A}$ - level) and 17 at a very low level $(\mathrm{S}+, \mathrm{S}-\mathrm{or} \mathrm{P}$ level). Specifically, moderate-to-high credibility of results showed little benefit on the risk of Alzheimer's disease from acetycholinesterase inhibitors, antihypertensive agents in late life, oestrogen therapy, and DHA+EPA supplementation. No robust conclusion could be reached for non-steroidal anti-inflammatory drugs, ginkgo biloba, cocoa flavanol and cognitive training. For directness of outcomes, five meta-analyses examined the associations with $A D$ (figure $4 B$ ). Although none showed a good performance in all the above domains, two interventions (physical exercise and total homocysteine-lowering treatment) seem more promising than others (figure 4C).

$\mathrm{A}+$ level), three were rated at a low-to-moderate level $(\mathrm{A}+/ \mathrm{A}-$ or $\mathrm{A}-$ level) and 17 were rated at a very low level $(\mathrm{S}+, \mathrm{S}-$ or P level) (figure 4B). The overall evaluation highlighted two interventions that seemed promising (figure 4C): physical exercise (mini-mental state examination (MMSE), standardised mean difference (SMD) $0.28,95 \% \mathrm{CI} 0.07$ to 0.50 and $\mathrm{AD}$ assessment scale cognition, SMD $0.25,95 \%$ CI 0.08 to 0.41 ) and tHcy-lowering treatment (MMSE, SMD 0.09, 95\% CI 0.02 to 0.15 ) (online supplementary appendix figure 3 ). Notably, oestrogen therapy was associated with an increase in the risk of dementia (G level).

The significance and the effect size minimally changed for most factors after excluding ORs (online supplementary appendix figure 4). No influences of publication bias on the pooled results were identified (online supplementary appendix 5). The sources of heterogeneity were explored. For diabetes $\left(n=14, I^{2}=65 \%\right)$, the percentage of women explained 39\% heterogeneity $(\mathrm{p}=0.008)$, which might be attributed to inclusion of two high-risk-of-bias studies $^{24} 25$ that explored associations only for men. The mean age at baseline explained most heterogeneity for hypertension $(p=0.0003)$ and BMI $\left(p=0.091, \tau^{2}=0\right)$. No influences of lowering the heterogeneity $\left(\mathrm{I}^{2}<10 \%\right)$ via sensitivity analyses on the pooled results were found for current smoking, systolic blood pressure, education and depression. The influence of risk of bias might be low for depression while smoking and stroke were vulnerable to sources of bias due to misclassification, attrition and generalisability (online supplementary appendix figure 5).

Twenty-one evidence-based suggestions with different levels of evidence (11 with Level A and 10 with Level B) and strength of suggestions (19 with Class I and two with Class III) are listed in table 1. Specifically, Class I suggestions were for 19 factors, including 10 factors with Level A evidence (cognitive activity, hyperhomocysteinaemia, increased BMI in late life, depression, stress, diabetes, head trauma, hypertension in midlife, orthostatic hypotension and education) and nine factors with Level $\mathrm{B}$ evidence (obesity in midlife, weight loss in late life, physical exercise, smoking, sleep, CVD, frailty, atrial fibrillation and vitamin C) (figure 5). Two factors were not recommended (Class III): oestrogen replacement therapy (Level A) and acetylcholinesterase inhibitors (Level B) (online supplementary appendix $7 \&$ appendix figure 6). Six factors (diastolic blood pressure management, NSAID use, social activity, osteoporosis, pesticide exposure and silicon from drinking water) were rated as Level $\mathrm{C}$ low-strength evidence, with the recommendation that their relationships with $\mathrm{AD}$ be confirmed in future studies.

\section{DISCUSSION}

Our systematic review and meta-analysis identified a total of 21 evidence-based suggestions that can be used in life-course 
Table 1 Guideline for prevention of AD: preliminary clinical suggestions *

\begin{tabular}{|c|c|}
\hline Factors/interventions & Suggestion \\
\hline \multicolumn{2}{|l|}{ Lifestyle } \\
\hline $\begin{array}{l}\text { BMI and weight } \\
\text { management }\end{array}$ & $\begin{array}{l}\text { Adults aged }<65 \text { years should maintain or lose weight through an appropriate balance of physical activity, caloric intake and formal behavioural programme } \\
\text { when indicated to maintain/achieve a BMI between } 18.5 \text { and } 24.9 \mathrm{~kg} / \mathrm{m}^{2} \text { (Class I, level } B \text { ) } \\
\text { Adults aged }>65 \text { years should not to be too skinny (Class I, level } A 4 \text { ) } \\
\text { Adults aged }>65 \text { years with a trend of weight loss should be closely monitored for their cognitive status (Class I, Leve/ B) }\end{array}$ \\
\hline Physical exercise & - Individuals, especially those aged $\geq 65$ years, should stick to regular physical exercise (Class I, Level $B^{*}$ ) \\
\hline Cognitive activity & - Mentally stimulating activities should be encouraged, such as reading, playing chess, etc (Class I, Level A4) \\
\hline Smoking & $\begin{array}{l}\text { People should not smoke and should avoid environmental tobacco smoke. Ccounselling, nicotine replacement and other pharmacotherapy as indicated } \\
\text { should be provided in conjunction with a behavioural programme or formal smoking cessation programme (Class I, Level B) }\end{array}$ \\
\hline Sleep & - Get sufficient and good quality sleep and consult a doctor or receive treatment when you have problem with sleep (Class I, Level B) \\
\hline \multicolumn{2}{|l|}{ Comorbidities } \\
\hline Diabetes & - Stay away from diabetes via a healthier lifestyle and diabetic patients should be closely monitored for their cognitive decline (Class I, Level A4) \\
\hline CVD & $\begin{array}{l}\text { Maintain a good condition of the cerebral vessels via a healthier lifestyle or medications to avoid atherosclerosis, low cerebral perfusion and any CVD. } \\
\text { Individuals with stroke, especially cerebral microbleeding, should be carefully monitored for their cognitive change and take preventative measures as } \\
\text { indicated to protect cognition (Class I, level B) }\end{array}$ \\
\hline Head trauma & D Protect your head from injuries (Class I, level A4) \\
\hline Frailty & - Stay healthy and strong in late life. Those with increasing frailty should be especially monitored for their cognition (Class I, Level B) \\
\hline Blood pressure & $\begin{array}{l}\text { Individuals aged }<65 \text { years should avoid hypertension via a healthier lifestyle (Class I, Level A4) } \\
\text { Individuals with } \mathrm{OH} \text { should be closely monitored for their cognition (Class I, Level A4) }\end{array}$ \\
\hline Depression & - Maintain a good condition of mental health and closely keep an eye on the cognitive status for those with depressive symptoms (Class I, Level A4) \\
\hline $\mathrm{AF}$ & - Maintain a good cardiovascular condition and manage AF using pharmaceuticals (Class I, level B) \\
\hline Stress & - Relax your mind and avoid daily stress (Class I, Level A4) \\
\hline \multicolumn{2}{|l|}{ Other domains } \\
\hline Education & Receive as much education as possible in early life (Class I, level A4) \\
\hline Hyperhomocysteinaemia & $\begin{array}{l}\text { Have a regular blood examination for homocysteine level. Individuals with hyperhomocysteinaemia should be treated with vitamin B and/or folic acid and be } \\
\text { followed with a focus on their cognition (Class I, Level A2) }\end{array}$ \\
\hline Vitamin C & V Vitamin C in the diet or taken as supplements might help (Class I, Level B) \\
\hline \multicolumn{2}{|l|}{ Not recommended } \\
\hline ERT & Oestrogen replacement therapy should not be specifically used for AD prevention in postmenopausal women (Class III, Level A2) \\
\hline $\mathrm{ACl}$ & - ACl should not be used for AD prevention in cognitively impaired individuals (Class III, Level B) \\
\hline
\end{tabular}

$\mathrm{ACl}$, acetylcholinesterase inhibitors; AF, atrial fibrillation; BMI, body mass index; CVD, cerebrovascular disease; ERT, estrogen replacement therapy; IMT, intima-media thickness; NSAIDs, nonsteroidal anti-inflammatory drugs; $\mathrm{OH}$, orthostatic hypotension.

practices to prevent $\mathrm{AD}$. Nineteen were regarded as 'strong suggestions', nine of which were rated with Level A evidence (table 1). Nearly two-thirds of these suggestions target vascular risk factors and lifestyle, strengthening the importance of keeping a good vascular condition and maintaining a healthy lifestyle for preventing AD.

\section{Strengths and weaknesses of this study}

This is the most comprehensive and large-scale systematic review and meta-analysis for $\mathrm{AD}$ prevention to date. The evidencebased suggestions are constructed by integrating a large amount of evidence from both OPSs and RCTs. Sources of bias and robustness of evidence were thoroughly assessed and secondary analyses were used to explore their influences, guaranteeing the objectivity and transparency of our findings. Furthermore, the outcome of OPSs was confined to AD dementia, given that the heterogeneity of endpoints might complicate the profile and downgrade the credibility of the evidence because: (1) observational studies are more vulnerable to sources of bias than RCTs, even though a rigorous procedure was employed to grade the evidence; (2) non-AD dementia accounts for roughly $30 \%$ of incident dementia (online supplementary appendix figure 7) and the false positive rate for diagnosis of mild cognitive impairment is fairly common. ${ }^{26}$ Some caveats should also be emphasised. Observational studies cannot indicate causal relationships and RCTs may not be generalisable beyond the specific sample, intervention, dose and duration studied. Classification of the available evidence including assessment of potential biases requires subjective judgement. The values of the current suggestions might be confined by geographic variability, definition of exposure and prevalence of risk factors at the population level. Some important factors of all-cause dementia were inadequately investigated for $\mathrm{AD}$, such as social determinants ${ }^{27}$ and frailty, ${ }^{28}$ and more high-quality prospective studies are warranted to bridge this gap. AD is challenging to study. The neurobiology of $\mathrm{AD}$ begins at least 15 years before symptoms appear. Tools such as amyloid and tau PET scanning are available to characterise the neuropathology at any stage, but it is impractical to include such assessments in large observational studies; without biomarker data, misclassification is unavoidable and several conclusions may be challenged by studies in the near future. Despite these challenges, this systematic review and meta-analysis can suggest recommendations to guide clinicians, even as the field perseveres with additional studies. These evidence-based suggestions must be presented in the context of the limitations of the studies and continuing uncertainty among investigators. Finally, the present study did not register and the protocol can be found in online supplementary appendix 8 .

\section{Strengths and weaknesses in relation to other studies}

Notably, tHcy-lowering treatment seems the most promising intervention for $\mathrm{AD}$ prevention, in agreement with a recent 


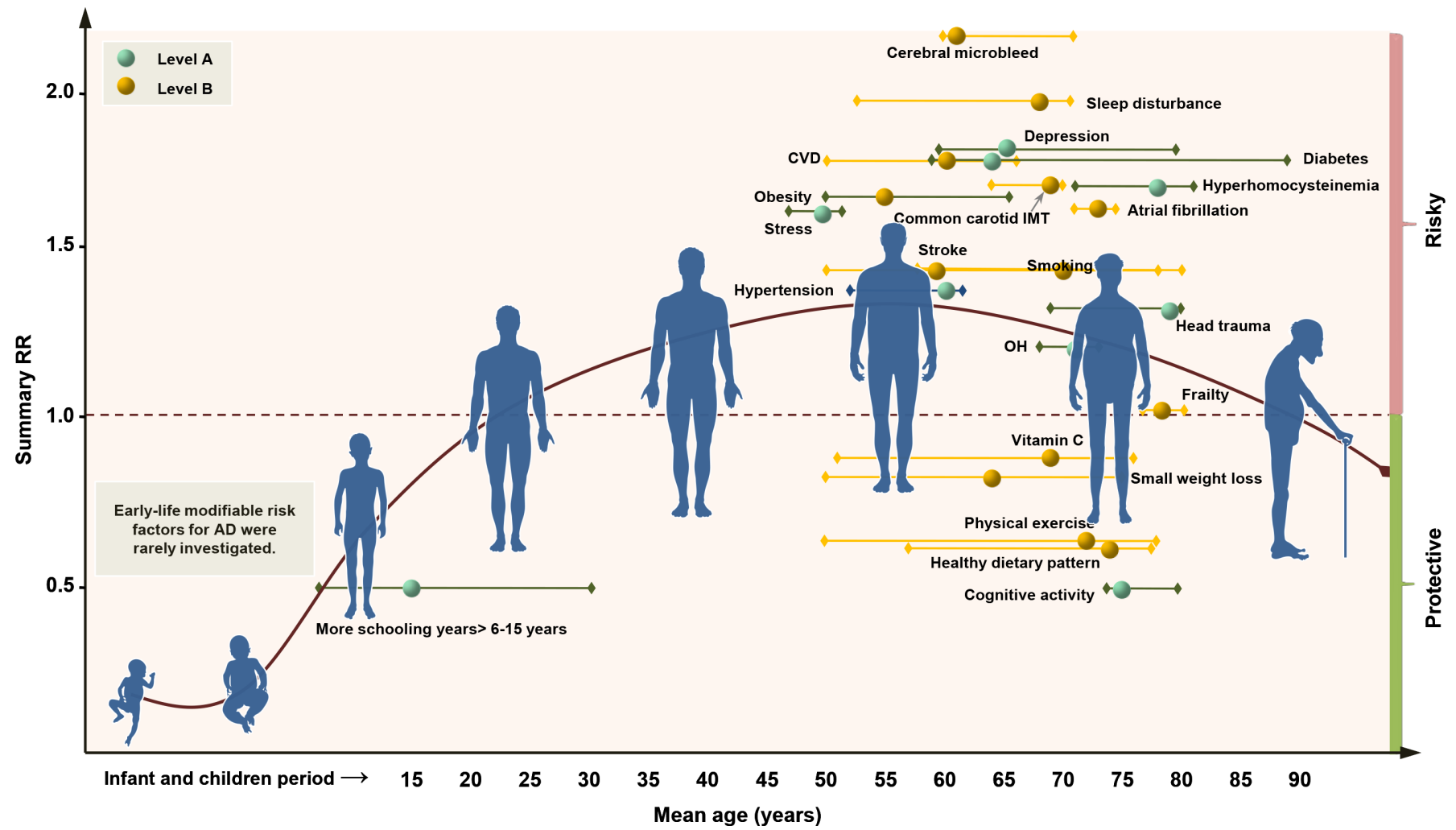

Figure 5 Distribution of modifiable factors with Class I recommendation throughout the course of life. Class I suggestions (benefit $>>$ risk due to intervention) risk factors include 10 factors with Level A evidence (cognitive activity, hyperhomocysteinaemia, increased BMI in late life, depression, stress, diabetes, head trauma, hypertension in midlife, orthostatic hypotension and education) and 9 factors (obesity in midlife, weight loss in late life, physical exercise, smoking, sleep, CVD, frailty, atrial fibrillation and vitamin C) with Level B evidence. The $x$ axis represents the mean age of the total sample (solid circle) with a range of mean age (short horizontal line) for observational prospective studies included. The y axis represents the summary relative risk (RR). AD, Alzheimer's disease; OH, orthostatic hypotension; CVD, cerebrovascular disease; IMT, intima-media thickness.

report. $^{29}$ The Lancet Commission on dementia has recently proposed nine potentially modifiable risk factors of all-cause dementia. However, these suggestions might not be directly applicable to $\mathrm{AD}$, bearing in mind that the heterogeneity of endpoints complicates the profile and reduces the credibility of evidence for $\mathrm{AD}$ prevention. Our study generated more evidence-based suggestions associated with a decreased risk of $\mathrm{AD}$, filling this gap in the field.

\section{Meaning of the study}

The hypotheses for the underlying mechanisms may include brain reserve theory, the hypoperfusion hypothesis, one-carbon methabolism, hypomethylation theory, inflammation and the oxidative stress hypothesis. The combination of multiple recommendations is most likely the best approach to delay the onset of $\mathrm{AD}$, as indicated by the Finnish Geriatric Intervention Study to Prevent Cognitive Impairment and Disability (FINGER). ${ }^{30}$ On the basis of this paper, future clinical trials should focus on exploring the best combination of recommendations with Class I recommendation and Level A evidence to prevent AD using larger samples, particularly in real-world settings. These evidence-based suggestions should be particularly noted by nondemented but high-risk individuals (eg, people with AOPE\&4, a high polygenic score, a family history of dementia or amyloidpositive evidence ${ }^{31}$ ) and family doctors to give optimal recommendations to their patients in terms of what they might do to get the best protection against $\mathrm{AD}$.

\section{Future research}

For OPSs, low participation rates (cognitive activity and stroke), high attrition (stroke, smoking, alcohol drinking and hypertension) and follow-up insufficiency (stroke and smoking) should be specifically highlighted in future prospective studies. Reverse causality might bias the association with late life obesity. ${ }^{32}$ It is unclear whether reverse causality exists for other potential factors such as frailty, social isolation and sleep disorders. Investigation and comparison of important characteristics of those who refused to participate or were lost during follow-up might be a good method to guarantee optimised validity. Subgroup effects exist due to the characteristics of the sample (eg, age, gender, ${ }^{33}$ $A P O E \& 4$ status $^{34}$ and medication compliance ${ }^{34}$ ) or exposure (eg, type, dose and duration). For RCTs, choosing the suitable population might be the key to determining whether an intervention can work. The optimal time window also matters, ${ }^{35}$ especially considering that benefits were weak for those with a clinical diagnosis of dementia. ${ }^{36}$ Generalisablity should be further optimised, such as recruiting larger samples from community-dwelling individuals and searching for methods to lower dropout rates. Welldesigned clinical trials are needed to verify the effects on AD of several promising interventions, including sleep improvement, smoking cessation, antidepression management and antidiabetic agents.

\section{CONCLUSIONS}

Twenty-one clinical evidence-based suggestions are proposed, offering clinicians and stakeholders an evidence-based guideline 
for $\mathrm{AD}$ prevention. With credible though inconclusive evidence, the suggestions targeted 10 risk factors including diabetes, hyperhomocysteinaemia, poor BMI management, reduced education, hypertension in midlife, orthostatic hypotension, head trauma, less cognitive activity, stress and depression. This study provides an advanced and contemporary survey of the evidence, suggesting that more high-quality OPSs and RCTs are urgently needed to strengthen the evidence base for uncovering more promising approaches to preventing AD.

\section{Author affiliations}

'Department of Neurology and Institute of Neurology, Huashan Hospital, Shanghai Medical College, Fudan University, Shanghai, China

2Department of Neurology, Qingdao Municipal Hospital, Qingdao University, Qingdao, China

${ }^{3}$ Department of Epidemiology and Public Health, University of Toulouse III, Toulouse, France

${ }^{4}$ Department of Psychiatry, Medical Research Council and Wellcome Trust

Behavioural and Clinical Neuroscience Institute, University of Cambridge, Cambridge, UK

${ }^{5}$ Department of Hygiene and Epidemiology, University of Ioannina Medical School, loannina, Greece

${ }^{6}$ Department of Epidemiology and Biostatistics, School of Public Health, Tongji Medical College, Huazhong University of Science and Technology, Wuhan, China ${ }^{7}$ Department of Neurology, Massachusetts General Hospital and Harvard Medical School, Charlestown, Massachusetts, USA

${ }^{8}$ Department of Neurology, Xuan Wu Hospital, Capital Medical University, Beijing, China

${ }^{9}$ Department of Psychological Medicine, Yong Loo Lin School of Medicine, National University of Singapore, Singapore

${ }^{10}$ Department of Neurology, Daping Hospital, Third Military Medical University, Chongqing, China

${ }^{11}$ Department of Medicine and Therapeutics, Faculty of Medicine, The Chinese University of Hong Kong, Shatin, Hong Kong

${ }^{12}$ Department of Neurology, University Hospital of Montpellier, Montpellier, France

${ }^{13}$ McGill Center for Studies in Aging, McGill University, Montreal, Quebec, Canada

${ }^{14}$ Alzheimer's Therapeutic Research Institute, University of Southern California, San Diego, California, USA

${ }^{15}$ Department of Geriatrics, Purpan University Hospital, Toulouse, France

\section{Twitter Wei Xu @na and Can Zhang @martindragoncn}

Acknowledgements The authors thank Professor Michael M Weiner and Dr YuTzu Wu for critical review.

Contributors JTY and BV conceived and designed the study. JTY, WX, CCT, HFW, MST and JQL selected the articles and extracted the data. JTY, WX, C-CT, HFW, M-ST, J-QL, XHH, YW, LT and LT proofread the data. JTY, WX, C-CT and AP analysed the data. WX and CCT generated the figures. JTY, WX, CCT wrote the first draft of the manuscript. JTY, WX, C-CT, SA, JS, EE, CZ, JJ, AP, LF, EHK, YJW, VM, JT, GS, PSA, QD, and $B V$ interpreted the data and contributed to the writing of the final version of the manuscript. All authors agreed with the results and conclusions and approved the final draft.

Funding This study was supported by grants from the National Key R\&D Program of China (2018YFC1314702), Shanghai Municipal Science and Technology Major Project (No. 2018SHZDZX03) and ZHANGJIANG LAB.

Competing interests JTY serves as an associate editor-in-chief for Annals of Translational Medicineand is senior editor for Journal of Alzheimer's Disease. SA has received grants from Europe, Ipsen, and France Alzheimer, served as a consultant for Ipsen, Pierre Fabre, Lilly, Nestlé, Sanofi and Servier, and received non-financial support from Biogen, Nutrition Santé, Pfzer and Icon, and other support from the AMPA Association. GS has received clinical trial support from Lilly and Roche in DIAN-TU, TauRx Therapeutics (TauRx) and Lundbeck; has been a data safety monitoring board (DSMB) member of ADCS, ATRI, API and Eisai; and has been a scientific adviser to Affiris, Boehringer Ingelheim, Lilly, Roche, Servier, Sanofi, Schwabe, Takeda and TauRx. PSA has received grants from the US Alzheimer's Association, Janssen, Lilly, the US National Institute on Aging and Toyama; and consulting fees from Abbott, Abbvie, Amgen, Anavex, AstraZeneca, Biogen Idec, Biotie, Bristol-Myers Squibb, Cardeus, Cohbar, Eisai, Elan, Eli Lilly, Genentech, Ichor, iPerian, Janssen, Lundbeck, Medivation, Merck, NeuroPhage, Novartis, Pfizer, Probiodrug, Roche, Somaxon and Toyama, outside the submitted work. BV reports grants from Pierre Fabre, Avid, Exonhit, AbbVie, Lilly, Lundbeck, MSD, Otsuka, Regenron, Sanofi, Roche, AstraZeneca, LPG Systems, Nestlé and Alzheon, and personal fees from Lilly, Lundbeck, MSD, Otsuka, Roche, Sanofi, Biogen, Nestlé, Transition Therapeutics and Takeda.
Patient consent for publication Not required.

Provenance and peer review Not commissioned; externally peer reviewed.

Open access This is an open access article distributed in accordance with the Creative Commons Attribution Non Commercial (CC BY-NC 4.0) license, which permits others to distribute, remix, adapt, build upon this work non-commercially, and license their derivative works on different terms, provided the original work is properly cited, appropriate credit is given, any changes made indicated, and the use is non-commercial. See: http://creativecommons.org/licenses/by-nc/4.0/.

\section{ORCID iDs}

Jin-Tai Yu http://orcid.org/0000-0002-7686-0547

Wei Xu http://orcid.org/0000-0002-3310-5875

\section{REFERENCES}

1 Qiu C, von Strauss E, Bäckman L, et al. Twenty-year changes in dementia occurrence suggest decreasing incidence in central Stockholm, Sweden. Neurology 2013:80:1888-94.

2 Matthews FE, Arthur A, Barnes LE, et al. A two-decade comparison of prevalence of dementia in individuals aged 65 years and older from three geographical areas of England: results of the Cognitive Function and Ageing Study I and II. Lancet 2013:382:1405-12.

3 Satizabal CL, Beiser AS, Chouraki V, et al. Incidence of dementia over three decades in the Framingham Heart Study. N Engl J Med 2016;374:523-32.

4 Wu Y-T, Fratiglioni L, Matthews FE, et al. Dementia in Western Europe: epidemiological evidence and implications for policy making. Lancet Neurol 2016;15:116-24.

5 Andrieu S, Coley N, Lovestone S, et al. Prevention of sporadic Alzheimer's disease: lessons learned from clinical trials and future directions. Lancet Neurol 2015:14:926-44.

6 Livingston G, Sommerlad A, Orgeta V, et al. Dementia prevention, intervention, and care Lancet 2017:390:2673-734.

7 Winblad B, Amouyel P, Andrieu S, et al. Defeating Alzheimer's disease and other dementias: a priority for European science and society. Lancet Neurol 2016;15:455-532.

8 Mazure CM, Swendsen J. Sex differences in Alzheimer's disease and other dementias. Lancet Neurol 2016:15:451-2.

9 Coley N, Andrieu S, Gardette V, et al. Dementia prevention: methodological explanations for inconsistent results. Epidemiol Rev 2008;30:35-66.

10 Moher D, Liberati A, Tetzlaff J, et al. Preferred reporting items for systematic reviews and meta-analyses: the PRISMA statement. Int J Surg 2010;8:336-41.

11 Stroup DF, Berlin JA, Morton SC, et al. Meta-analysis of observational studies in epidemiology: a proposal for reporting. Meta-analysis Of Observational Studies in Epidemiology (MOOSE) group. JAMA 2000;283:2008-12.

12 Weuve JMM, Blacker D. The AlzRisk database. Alzheimer research forum. Available: http://www.alzforum.org [Accessed [date of access]

13 McKhann G, Drachman D, Folstein M, et al. Clinical diagnosis of Alzheimer's disease: report of the NINCDS-ADRDA Work Group under the auspices of Department of Health and Human Services Task Force on Alzheimer's Disease. Neurology 1984:34:939-44

14 . Available: http://community.cochrane.org/editorial-and-publishing-policy-resource/ cochrane-review-development/cochrane-handbooks/cochrane-handbook-systematic reviews-interventions

15 Deeks JJ, Dinnes J, D'Amico R, et al. Evaluating non-randomised intervention studies. Health Technol Assess 2003; 7:27.

16 Guyatt GH, Oxman AD, Vist G, et al. GRADE guidelines: 4. Rating the quality of evidence--study limitations (risk of bias). J Clin Epidemio/ 2011;64:407-15.

17 Guyatt GH, Oxman AD, Kunz R, et al. GRADE guidelines: 7. Rating the quality of evidence--inconsistency. J Clin Epidemiol 2011;64:1294-302.

18 Guyatt GH, Oxman AD, Kunz R, et al. GRADE guidelines 6 . Rating the quality of evidence--imprecision. J Clin Epidemiol 2011;64:1283-93.

19 Higgins JPT, Thompson SG, Deeks JJ, et al. Measuring inconsistency in meta-analyses. BMJ 2003:327:557-60.

20 Riley RD, Higgins JPT, Deeks JJ. Interpretation of random effects meta-analyses. BMJ 2011:342:d549.

21 Higgins JPT, Thompson SG. Quantifying heterogeneity in a meta-analysis. Stat Med 2002;21:1539-58.

22 Egger M, Davey Smith G, Schneider M, et al. Bias in meta-analysis detected by a simple, graphical test. BMJ 1997;315:629-34.

23 Peters JL, Sutton AJ, Jones DR, et al. Contour-enhanced meta-analysis funnel plots help distinguish publication bias from other causes of asymmetry. J Clin Epidemiol 2008:61:991-6.

24 Moffat SD, Zonderman AB, Metter EJ, et al. Free testosterone and risk for Alzheimer disease in older men. Neurology 2004;62:188-93.

25 Dal Forno G, Palermo MT, Donohue JE, et al. Depressive symptoms, sex, and risk for Alzheimer's disease. Ann Neurol 2005:57:381-7.

26 Koepsell TD, Monsell SE. Reversion from mild cognitive impairment to normal or nearnormal cognition: risk factors and prognosis. Neurology 2012;79:1591-8. 
27 Fratiglioni L, Wang HX, Ericsson $\mathrm{K}$, et al. Influence of social network on occurrence of dementia: a community-based longitudinal study. Lancet 2000;355:1315-9.

28 Stower H. Linking Alzheimer's disease and frailty. Nat Med 2019;25:358.

29 Kane RL, Butler M, Fink HA, et al. Interventions to prevent age-related cognitive decline, mild cognitive impairment, and clinical Alzheimer's-type dementia. Rockville (MD, 2017.

30 Ngandu T, Lehtisalo J, Solomon A, et al. A 2 year multidomain intervention of diet, exercise, cognitive training, and vascular risk monitoring versus control to prevent cognitive decline in at-risk elderly people (FINGER): a randomised controlled trial. Lancet 2015;385:2255-63.

31 Donohue MC, Sperling RA, Petersen R, et al. Association between elevated brain amyloid and subsequent cognitive decline among cognitively normal persons. JAMA $2017: 317: 2305-16$
32 Kivimäki M, Luukkonen R, Batty GD, et al. Body mass index and risk of dementia: analysis of individual-level data from 1.3 million individuals. Alzheimers Dement 2018;14:601-9.

33 Zissimopoulos JM, Barthold D, Brinton RD, et al. Sex and race differences in the association between statin use and the incidence of Alzheimer disease. JAMA Neurol 2017;74:225-32.

34 Rajan KB, Barnes LL, Wilson RS, et al. BP and risk of incident AD dementia by antihypertensive and APOE epsilon4 allele. Ann Neurol 2018;83:935-44.

35 Kryscio RJ. Secondary prevention trials in Alzheimer disease: the challenge of identifying a meaningful end point. JAMA Neurol 2014;71:947-9.

36 Lamb SE, Sheehan B, Atherton N, et al. Dementia and physical activity (DAPA) trial of moderate to high intensity exercise training for people with dementia: randomised controlled trial. BMJ 2018:361:k1675. 